\title{
Analysis of platelet function: role of microfluidics and nanodevices
}

\author{
María José Santos-Martínez, ${ }^{* a b c}$ Adriele Prina-Mello, ${ }^{b c}$ Carlos Medina ${ }^{a}$ and Marek Witold Radomski ${ }^{a c}$ \\ Received 31st May 2011, Accepted 27th September 2011 \\ DOI: $10.1039 / \mathrm{c} 1 \mathrm{an} 15445 \mathrm{a}$
}

\begin{abstract}
Platelet aggregation is essential for vascular haemostasis and thrombosis. To improve the therapy of arterial thrombotic disorders and identify novel therapeutic targets it is imperative to study basic mechanisms of platelet thrombus formation. To date most data on biology, physiology and pharmacology of platelet aggregation have been obtained by studying this phenomenon under static or quasi-dynamic conditions at the macroscale level. There is a widespread recognition for the need of new technologies that will help to further elucidate the role of platelets in physiological and pathological thrombus formation and to design more effective and specific antithrombotic drugs. Micro- and nanofluidic devices, capable of reaching nanoscale resolution, can be used for this purpose setting the scene for the development of novel methods for studying platelet function in physiology, pathology and therapeutics.
\end{abstract}

\section{Introduction}

Platelets are anucleate cell elements derived from megakaryocytes that play a crucial role in haemostasis and thrombosis.

${ }^{a}$ School of Pharmacy and Pharmaceutical Sciences, Panoz Institute, Trinity College Dublin, Dublin 2, Ireland. E-mail: santosmm@tcd.ie; Fax: +353-1-8963367; Tel: +353-1-8964281

${ }^{b}$ School of Medicine, Trinity College Dublin, Dublin 2, Ireland

${ }^{c}$ Centre for Research on Adaptive Nanostructures and Nanodevices, Trinity College Dublin, Dublin 2, Ireland
Human platelets are discoid, 2-4 $\mu \mathrm{m}$ in size and contain numerous alpha $(\alpha)$ and dense $(\delta)$ granules, which store factors and platelet receptor proteins that are crucial for platelet function. In the absence of any activating stimulus, platelets circulate in a quiescent state. Endothelial cell-released factors such as nitric oxide (NO) and prostaglandin $\mathrm{I}_{2}\left(\mathrm{PGI}_{2}\right)$ and $\mathrm{CD} 39$, an adenosine diphosphate (ADP)-ase, present on the surface of endothelial cells, are inhibitory factors that control platelet activation. When the vascular wall is damaged, due to the loss of the endothelial cell barrier or its physiological function,

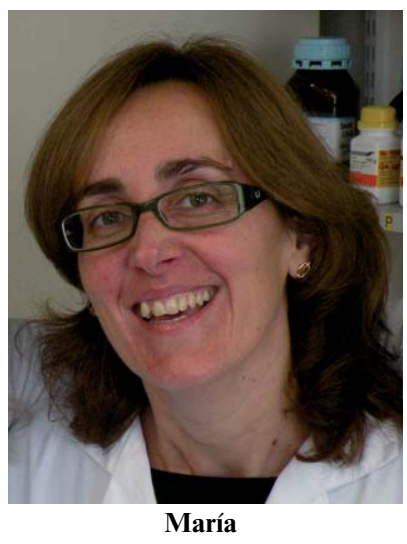

José Santos-Martínez
Dr Santos-Martinez is a Spanish medical doctor specialist in Respiratory Medicine. She started her formation as a basic researcher in 2004 at Institute of Molecular Medicine for the Prevention of Human Diseases in Houston-Texas, USA. In 2006 she moved to Ireland and in 2009 she defended her PhD in Pharmacology in Trinity College Dublin (TCD). In 2010, she was appointed as an Ussher Lecturer in Nanopharmaceutical Drug Discovery in a joined position between the School of Pharmacy and the School of Medicine in TCD. Her research is primarily focused on platelet biology, cancer, asthma and nanomedicine.

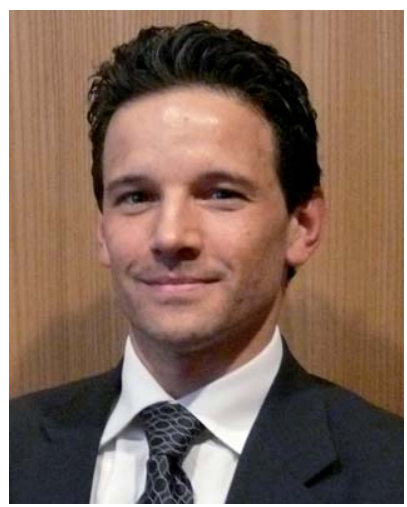

Adriele Prina-Mello
Dr Adriele Prina-Mello (MSc degree in Material Science and Engineering from Polytechnic of Turin (Italy) and PhD degree in Bioengineering and Nanobiotechnology from Trinity College Dublin (Ireland)) is a Senior Research Fellow of the School of Medicine, member of the TCD coordination team of a large FP7 project, and CRANN investigator (TCD). He is the Vice-Chairman of the Nanodiagnostic Working Group of the European Technology Platform in NanoMedicine. Dr Prina-Mello's research interests focus on advanced translation in NanoMedicine (diagnostic and imaging), dynamic interaction between nano-objects and biological models (nanotoxicology, nanobiocompatibility and nanobiotechnology), microfluidics, biomedical devices and tissue engineering applications of nanotechnology and nanomaterials. 
circulating platelets are recruited to the site of injury responding to this event in three consecutive and integrated phases that involve adhesion, activation and platelet aggregation. Initial binding and adhesion of platelets to the exposed subendothelium are mainly mediated by GPIb/IX/V complex and collagen receptors, GPVI and GPIa/IIa, in the platelet surface, and by von Willebrand factor (vWF) and fibrillar collagen in the vascular site. These adhesive interactions basically depend on the rheological conditions at the site of vascular damage. At low shear rate, platelet adhesion to the vessel wall primarily involves binding to fibrillar collagen, fibronectin and laminin. However, under high shear stress the initial adhesion of platelets to the subendothelium is mediated primarily through the interaction of vWF with the GPIb/IX/V complex, which is required to decelerate platelets and allow the platelet collagen receptors to bind to collagen and to firmly adhere to the surface. The binding of vWF to the GPIb/IX/X complex also leads to the release of platelet granules and activation of the GPIIb/IIIa receptor that is required to promote the interactions with adjacent platelets and fibrinogen. Activated platelets also express on their surface P-selectin mediating platelet-leukocyte aggregation. ${ }^{1-4}$ The recruitment of additional platelets is mediated by a variety of locally accumulating mediators such as Thromboxane $\mathrm{A}_{2}\left(\mathrm{TXA}_{2}\right){ }^{5} \mathrm{ADP}^{6}$ and matrix metalloproteinase-2 (MMP-2) ${ }^{7}$ that are secreted or released from activated platelets and thrombin, which is produced on the surface of activated platelets. These may result in haemostatic plug to repair the vascular damage that occurs with daily life, or lead to occlusive thrombus formation (Fig. 1).

\section{Conventional methods for the measurement of platelet function}

Platelet aggregation is one of the most important biological processes responsible for the maintenance of vascular haemostasis. However, the distance between physiological haemostasis

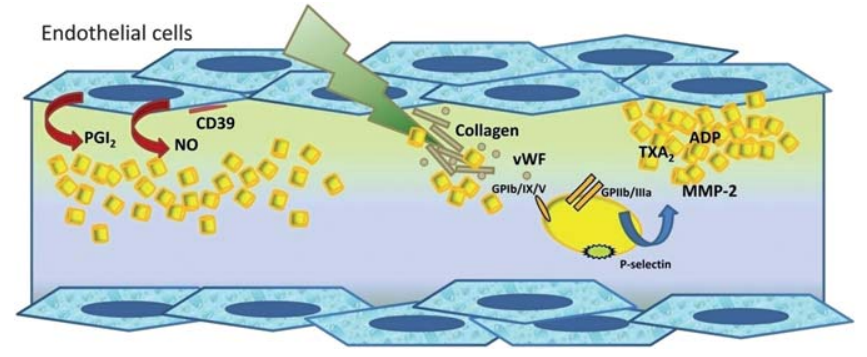

Fig. 1 A simplified overview of platelet plug formation. Platelets are maintained in the resting state by the combined action of inhibitory factors including prostaglandin $\mathrm{I}_{2}\left(\mathrm{PGI}_{2}\right)$, nitric oxide (NO) and CD39. The development of the platelet plug is initiated by the adhesion of collagen and von Willebrand factor (vWF) to platelet glycoprotein (GP) receptors. At the same time platelet receptor P-selectin translocates to the membrane surface and GPIIb/IIIa becomes activated. The platelet plug is extended as additional platelets are activated via the release or secretion of platelet agonists such as Thromboxane $\mathrm{A}_{2}\left(\mathrm{TXA}_{2}\right)$, adenosine diphosphate (ADP) and matrix metalloproteinase-2 (MMP-2). Finally, close contacts between platelets in the growing haemostatic plug, along with a fibrin network, help to maintain and stabilize the platelet aggregate. Platelets are represented as yellow structures.

and pathological thrombosis is very short and enhanced and inappropriate aggregation contributes to the pathophysiology of prevalent civilisation disorders such as ischemic heart disease and stroke. It is widely recognized that platelets are at least partially responsible for the pathological development of atherothrombosis, the leading cause of death in the developed world. ${ }^{8-10}$ Therefore, the underlying phenomenon of thrombus formation and the use of different anti-platelet drugs to prevent the development or recurrence of thrombotic diseases of the coronary and cerebrovascular circulation have been extensively studied during the last few decades.

There was little research in arterial thrombosis and relatively little understanding of platelet function before 1961 when O'Brien reported for the first time the use of a strong hand lens

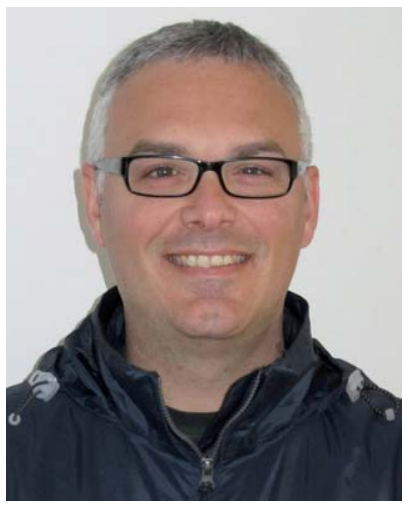

Carlos Medina
Dr Carlos Medina was born in Tenerife, Spain. After studying Medicine at University of La Laguna, he specialized in Gastroenterology at Vall d'Hebron Hospital, Barcelona. Since graduating with a PhD in Medicine from University of Barcelona in 2003, he held a research post-doctoral fellowship (MECD/Fulbright) at the University of Texas-Houston. In 2007 he was appointed as a Science Foundation Ireland Stokes lecturer in Pharmacology, School of Pharmacy,

TCD. Presently he leads a pharmacology research group supported by SFI and Enterprise Ireland. His research is primarily focused in two areas: (1) pharmacological treatments for intestinal diseases and (2) nanomedicine.

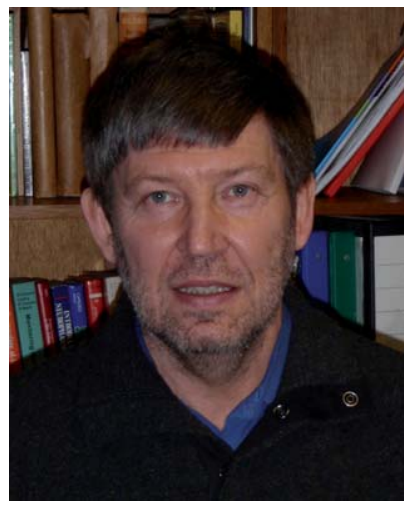

Marek Witold Radomski
Marek W. Radomski, $M D$, FTCD, DHC is a Professor of Pharmacology and Head of School of Pharmacy and Pharmaceutical Sciences Trinity College Dublin. He is a highly cited pharmacologist (www. isihighlycited.com) with longstanding interest in platelet biology and pharmacology. His research has been focused on the role of nitric oxide, matrix metalloproteinases and, more recently, nanomaterials in platelet regulation. 
with powerful cross-illumination to monitor aggregation in platelet-rich plasma. ${ }^{11}$ Later, Born developed a simple method for studying the response of platelets to platelet agonists. ${ }^{12}$ The method recorded changes in light transmission of a stirred platelet suspension exposed to a platelet agonist when platelet aggregates were forming. This device (commonly called a light aggregometer) exerted a profound effect on the study of platelet reactions, and it rapidly came into routine use worldwide ${ }^{13}$ (Fig. 2). Almost two decades later, another device to assess platelet function was developed. The new instrument, called an electronic aggregometer, was similar to a light aggregometer, but, rather than light transmission, it measured the increase in electrical impedance across two metal wires that results from platelet aggregation in anticoagulated whole blood.$^{14}$ Later on, a rapid platelet function analyzer (RPFA) was developed as a point-of-care instrument designed to measure the effects of antiplatelet therapy. It is a fully automated platelet aggregometer originally developed to monitor GPIIb/IIIa antagonists. ${ }^{15}$ It features three different cartridges to measure the level of inhibition of platelet aggregation achieved by aspirin, $\mathrm{P} 2 \mathrm{Y}_{12}$ ADP receptor antagonists or GPIIb/IIIa inhibitors, but not their combination. ${ }^{16}$

The light aggregometer has been considered as the historical gold standard of platelet function testing. In fact, it is possible to obtain a large amount of information about many different aspects of platelet function adding a set of agonists to stirred platelets. ${ }^{17}$ Because platelets are stirred to maintain them in suspension, platelet-platelet interactions in the aggregometer take place under the influence of fluid mechanical shear stress. However, the shear stress varies throughout the stirred platelet suspension and does not mimic flow and shear rates encountered

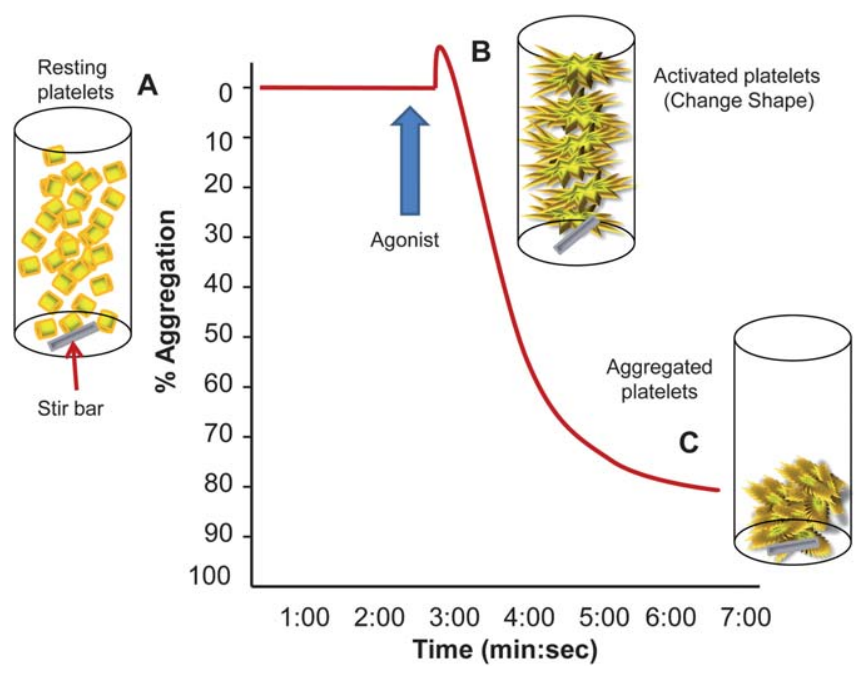

Fig. 2 Platelet function testing: light transmission aggregometry. The light aggregometer measures changes in light transmission of a stirred platelet suspension exposed to a platelet agonist. It is calibrated by a cuvette containing platelet poor plasma which equates to $100 \%$ light transmission. Platelet rich plasma stirred in the absence of platelet agonist equates to $0 \%$ light transmission (baseline, A). Change in platelet shape due to platelet activation induced by the addition of a platelet agonist results in a short decrease in the light transmission (B). When platelet aggregates are forming an increase in the light transmission is recorded by the device and calculated as percentage of aggregation (C). in arterial circulation. ${ }^{13}$ Moreover, platelets are stirred under low shear conditions and only form aggregates after addition of agonists, conditions which do not accurately mimic platelet adhesion, activation and aggregation upon vessel wall damage. ${ }^{17}$ In addition, the conventional light transmission methods quantifying platelet aggregation have not been sufficiently sensitive to detect micro-aggregates. ${ }^{18}$

A number of different devices have been also developed in order to mimic, as close as possible, the processes that take place during vessel wall damage. A cone and plate analyzer involves a cone rotating on a plate that generates a controlled shear stress (from less than 2 dynes $\mathrm{cm}^{-2}$ to greater than 200 dynes $\mathrm{cm}^{-2}$ ) allowing the study of the kinetics of aggregate formation. It is considered one of the instruments of choice (the another one is the coaxial cylinder Couette) for studying the effects of bulk shear stress on platelet function. ${ }^{19}$ In addition, the imaging of platelet adhesion and aggregation to the plate coated with collagen or extracellular matrix (ECM) can be also investigated ${ }^{16}$ (Fig. 3A). A platelet function analyzer 100 (PFA-100) creates an artificial vessel consisting of a sample reservoir, a capillary and a biologically active membrane with a central aperture (collagenepinephrine-coated or collagen-ADP-coated). The sample is aspirated from the reservoir at high shear rates $\left(5000-6000 \mathrm{~s}^{-1}\right)$ through the capillary and the aperture applying a negative pressure, mimicking in this way the resistance and the injured part of a small artery, and the time necessary for a platelet plug to occlude the aperture is monitored. ${ }^{20}$ However, it has been demonstrated that this method has limited sensitivity and specificity. ${ }^{21,22}$ A coaxial cylinder Couette device was originally invented for studies of viscosity and biological particle suspensions in controlled flow and later on adapted to measurements of platelet function. ${ }^{23}$ It consists of two coaxial cylindrical chambers separated by a gap where controlled rotation of the inner cylinder generates the desired shear rates (100-8000 s $\left.\mathrm{s}^{-1}\right)$. Using this device sampling of micro-volumes of platelets for further analysis such as flow cytometry is also possible ${ }^{24}$ (Fig. 3A).

In addition, in vitro studies of coagulation-dependent thrombus formation can be also performed using perfusion chamber devices. The most widely employed are the annular perfusion chambers and the parallel-plate perfusion chambers. ${ }^{16}$ An annular perfusion chamber, developed by Baumgartner, ${ }^{25}$ was the first successful and large-scale used in vitro device of thrombus formation. In this model, a segment of a vessel is everted and mounted centrally on a bar that is surrounded by an outer cylinder. By changing the flow rate or the distance between the subendothelial surface and the inner wall of the cylinder, the shear rate at the subendothelial surface can be varied from 50 to $3300 \mathrm{~s}^{-1}$ (ref. 26) (Fig. 3B). A Parallel plate perfusion chamber device was developed by Sakariassen. ${ }^{27}$ Similar to the annular chamber, it consists of a rectangular flow channel coated with components capable of inducing thrombus formation. It provides a controlled and well-defined flow environment. ${ }^{19}$ Modifying the flow rate, or the height and/or the width of the chamber, shear rates can range from 50 to $10500 \mathrm{~s}^{-1} .{ }^{26}$ A new generation of perfusion chambers or parallel-plate perfusion chamber devices with stenosis have been also developed in the last few decades. However, platelet function measurements using these flow-mimicking devices or point-of-care instruments have limited sensitivity and specificity and do not correlate well with 
A
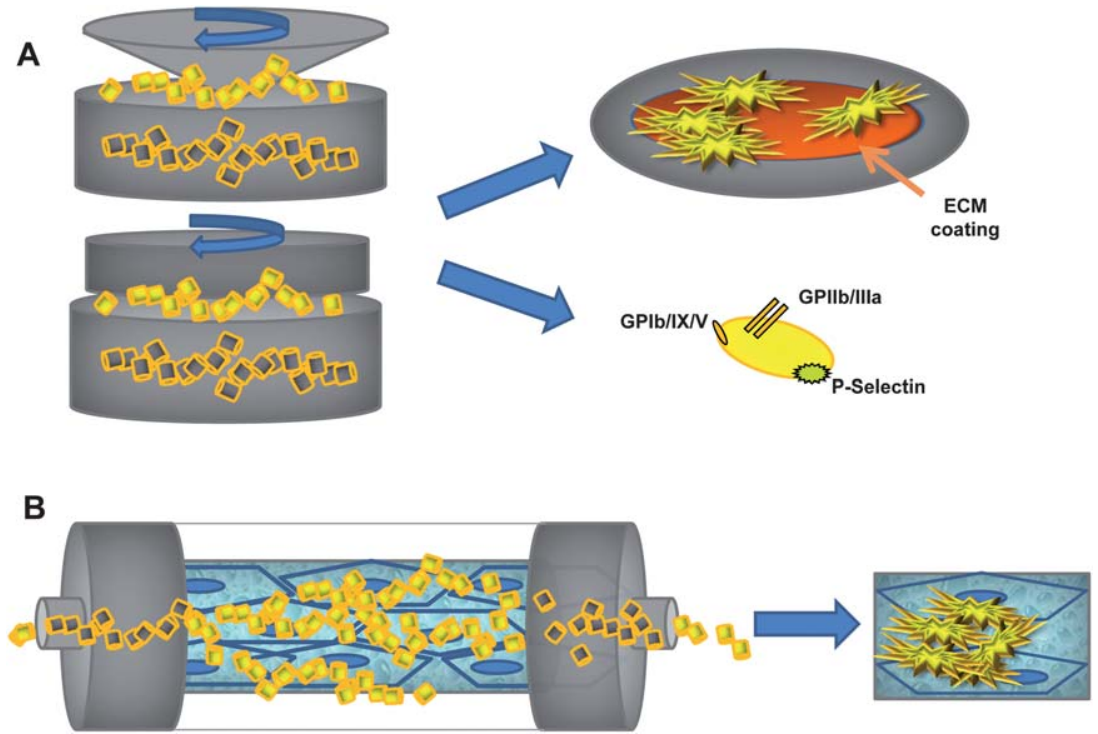

Fig. 3 Platelet function testing: devices for the study of thrombus formation. A cone and plate analyzer and coaxial cylinder Couette involve a cone/ cylinder that by rotating on a plate/chamber generates controlled shear stresses. Using these devices thrombus formation can be microscopically visualized and platelet activation studied by flow cytometry (A). Using an annular perfusion chamber (B) a segment of a vessel is mounted on a bar enclosed by a cylinder where the shear rate can be also modified.

the aggregometer that due to the lack of better methodology still stands as the "traditional gold standard" for the measurement of platelet aggregation.

\section{New technologies for the measurement of platelet function}

The devices described in the previous section are used for detection of platelet activation and monitoring the clinical effectiveness of flagship antiplatelet drugs such as aspirin or $\mathrm{P}^{2} \mathrm{Y}_{12}$ ADP receptor antagonists. ${ }^{28-30}$ However, it is really challenging to monitor the pharmacological effects of these drugs and to individualize treatments. This is mainly due to the fact that most of the method assays available to date are not sensitive enough to detect platelet microaggregation as they assay platelet activation-aggregation in macroscale. During the last few years the introduction of nanotechnology has made possible the development of novel custom-design microfluidic systems for the study of platelet size and morphology. Inglis et al. have used a device which constantly fractionates particles according to size by displacing them perpendicularly to the fluid flow direction in a micro-fabricated post-array. With this method, whole blood can be used and platelets detected using the platelet specific antibody anti-CD41 without the use of an activation specific label or marker. The authors suggest that the device may be useful in a wide range of medical procedures and diagnostics which involve platelets providing rapid diagnostic information about the haemostatic condition of a blood sample. ${ }^{31}$ Microfluidic devices have been also used to study the interactions between platelets and protein surfaces at physiological flowshear conditions with minimum amount of blood sample. Following this concept, Gutierrez et al. have constructed multichannel microfluidic devices that allow the study of platelet adhesion to extracellular matrix proteins at different physiological ranges of shear stresses with less than $100 \mu \mathrm{L}$ of blood. Using those microfluidic platforms, dynamic adhesion of human and murine platelets to $\mathrm{VWF}$, fibrinogen and collagen was studied using high-resolution fluorescence imaging. The role of the GPIIb/IIIa receptor (with wild-type mice and mice with impaired GPIIb/IIIa receptors) was also investigated. ${ }^{32}$ Neeves et al. have developed an in vitro microfluidic model of focal vascular injury using immobilized collagen. Using this method the response of murine platelet deposition to focal zones of prothrombotic stimuli was monitored by fluorescence microscopy. Platelet accumulation was measured, at equivalent to venous and arterial wall shear rates, using whole blood from wild-type mice and mice deficient in collagen receptors. Following the formation of the thrombus its stability was investigated by stepping-up the shear rate to $8000 \mathrm{~s}^{-1}$ in the presence or absence of murine PAR4 activating peptide. ${ }^{33}$ Sarvepalli et al. have designed and built another microfluidic chamber in which a wall shear rate of $1000 \mathrm{~s}^{-1}$ can be reached with a flow rate of $15 \mu \mathrm{L} \mathrm{min}{ }^{-1}$. Human platelet adhesion on albumin-collagen coated surfaces was described and characterized, at relevant arterial and venous shear rates, and compared with a standard parallel plate flow chamber. ${ }^{34}$ Schneider et al. using a microfluidic flow chamber incorporated on a chip were able to mimic a large range of blood flow conditions and directly visualize the conformational dynamics of vWF under shear flow, a protein which is essential for vascular haemostasis. In fact, they found that the application of extraordinarily high shear flows $\left(>1000 \mathrm{~s}^{-1}\right)$ resulted in a rapid elongation of vWF fibres in solution..$^{35}$ More recently Kent et al. reported the use of a miniature parallel-plate perfusion chamber to analyze platelet-vWF interactions under controlled shear-rates with approximately 150 $\mu \mathrm{L}$ of human blood. Using this method, the percentage coverage of platelets is quantified using a custom software algorithm. ${ }^{36}$ One of the first articles demonstrating the enabling application of 


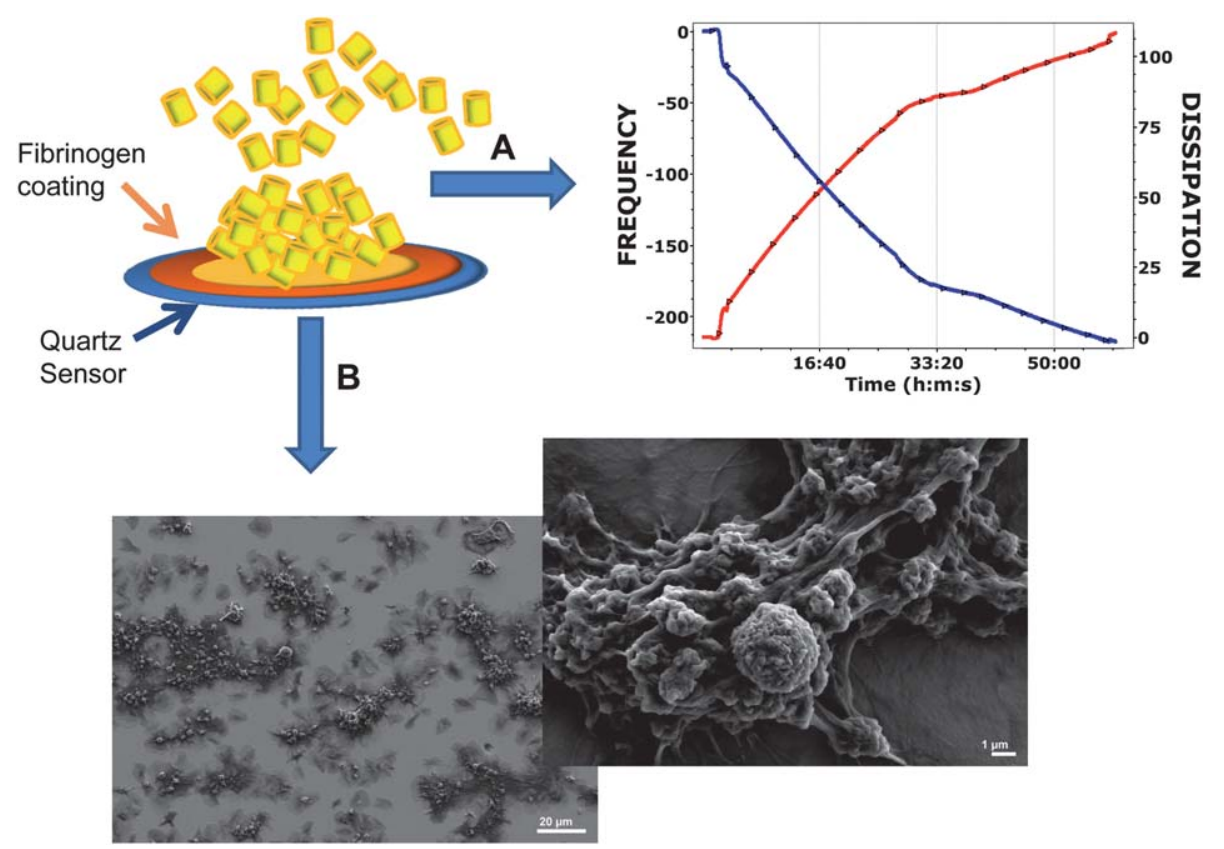

Fig. 4 Quartz Crystal Microbalance with Dissipation monitoring (QCM-D) for the study of platelet aggregation. Perfusion of platelet rich plasma through the device induces a decrease in $f$ (blue line, left axis) and an increase in $D$ (red line, right axis) measured at real time (A). The deposition of microaggregates on the sensor surface can be visualized using highly advanced microscopy, such as scanning electron microscopy (B).

a microfluidic device to conduct high-throughput signalling studies and drug discovery screening against human platelet targets was published by Tran et al. In that work, the time course of agonist-dependent calcium flux affected by platelet agonists on fluor-loaded human platelets was optimized in a macrosample cuvette format in preparation for detection in a microfluidic chip-based assay. ${ }^{37} \mathrm{Ku}$ et al. have also reported the use of a microfluidic device to study platelet adhesion to endothelium. To demonstrate the ability of platelets to adhere to an immobilized endothelium upon activation, platelets were incubated in solutions containing different concentrations of ADP and hydrodynamically pumped through an endothelium-lined microchannel. In order to demonstrate the utility of the microfluidic device for future studies involving drug discovery, platelets were activated with increasing concentrations of ADP in the presence and absence of the antiplatelet agent clopidogrel. It was found that in the presence of clopidogrel the number of platelets adhering to endothelium decreased significantly. ${ }^{38}$ Maloney et al. have reported the use of an eight-channel microfluidic device where the pharmacological effect of Apyrase and ATP and ADP receptor antagonists on thrombus formation was evaluated. In this study accumulation of fluorescent platelets was measured in the presence of the inhibitors and the $\mathrm{IC}_{50}$ curves calculated perfusing human whole blood at a uniform shear rate of $200 \mathrm{~s}^{-1}$ across the channels. ${ }^{39}$ Mimicking in vitro the effects of pathological changes in blood vessel geometry Tovar-Lopez et al. have recently designed a micro-device to monitor dynamic platelet aggregation in response to strain rate micro-gradients under flow conditions. The application of controlled strain rate microgradients in this device offers the ability to achieve periods of high strain rates with minimal sample volume and the ability to control the spatial location and extent of forming aggregates, making this device a suitable tool to elucidate the mechanisms underlying this shear-dependent process. ${ }^{40}$ We have also employed microfluidics for studying cancer cell-platelet interactions and showed that platelets have the ability to disrupt platelet-cancer cell aggregate and this phenomenon may contribute to blood-borne cancer metastasis. ${ }^{41}$ George et al. have also used microfluidics to investigate whether tumour-derived supernatant from melanoma and colon cancer may induce adhesion of circulating platelets to endothelial cells. They found that tumour-derived supernatant rapidly induced platelet-string formation via secreted vWF under flow conditions. ${ }^{42}$

Using the novel devices described above the quantification of platelet adhesion-aggregation is based on aggregate size using microscopy and/or mathematical models. The main advantage of those methods is the possibility to study shear-dependent platelet adhesion-aggregation with small sample volumes opening the field to the development of lab-on-a-chip research, point-of-care and high-throughput screening instruments under flow conditions. $^{43}$

Another interesting application for the study of platelet adhesion-aggregation phenomenon involves the use of sensor technologies adapted for biological-medical sciences. The principle of analysis of a Quartz Crystal Microbalance (QCM) is based on the resonance frequency of a quartz crystal sensor induced by applying an alternating electric field across the crystal. Deposition on the sensor surface induces a decrease in the frequency of vibration $f$ that is proportional to the mass. ${ }^{44}$ Following this approach Sinn et al. have recently reported the use of QCM to monitor platelet aggregation on fibrinogen-coated and uncoated gold sensors measuring changes in the $f$ under non-flow conditions. In this study platelet agonists and a GPIIb/IIIa receptor antagonist were also used to modulate platelet aggregation. ${ }^{45}$ Although similar devices have been used previously to detect platelet adhesion, ${ }^{46-48}$ this is the first time where the use of this 
technology is compared with the light aggregometer, the well established "gold standard" for the measurement of platelet aggregation. However, it is important to highlight that the deposition of a soft or thick layer on the sensor surface also induces a high dissipation shift $D$ that reveals the layer's elasticity and, in these situations, the mass can be underestimated by measuring only the $f$. In fact, it has been demonstrated that the combined measurement of $f$ and $D$ using a Quartz Crystal Microbalance with Dissipation monitoring (QCM-D) could be useful to explore cell-surface interactions for biomaterials. ${ }^{49-51}$ Based on these observations our group has developed a method for the quantification and pharmacological characterization of platelet aggregation under flow conditions using a QCM-D. We have shown that platelet micro-aggregation can be quantified and pharmacologically regulated under flow conditions in real time using a QCM-D. ${ }^{52,53}$ Furthermore, we have demonstrated that onsensor platelet adhesion and aggregation can be imaged with nanoscale resolution using atomic force microscopy. Interestingly, Weber et al. have investigated the binding kinetics of GPIIb/ IIIa to polymer-adsorbed fibrinogen using a QCM-D and correlated the results with platelet adhesion to the polymer surfaces by scanning electron microscopy. ${ }^{54}$ QCM-D has been also used to quantify platelet morphological changes by electron microscopy focusing on the platelet primary adhesion process on a mix of fibronectin and albumin deposited on silica crystals. ${ }^{55}$ More recently the same group has studied platelet activation on different protein layers on polymer-modified surfaces. The thrombogenic properties of the different surfaces were analyzed and compared by plotting $D$, as a function of corresponding $f$ and time, against the corresponding frequency shift. ${ }^{56} \mathrm{~A}$ QCM-D is a low shear rate and shear stress flow device that mimics the thrombotic events taking place in the microvasculature. The device is extremely sensitive, being capable of detecting platelet deposition in the presence of very low platelet numbers. ${ }^{52,53}$ In fact, due to its sensitivity, it could be a suitable device for studying platelet function under conditions such as thrombocytopenia or when the amount of blood available is limited such as in neonates. In addition, the sensor constitutes an excellent platform for the imaging of platelet nano-microaggregate formation using highly advanced microscopy (Fig. 4).

\section{Conclusion}

In recent years, the application of microfluids and nanodevices has made a substantial progress in the field of platelet biology and pharmacology. These methods can contribute to a better understanding of platelet adhesion and aggregation under flow conditions. In this review we highlight that nanoscale resolution micro- and nanofluidic devices can be used for the measurement of early stages of platelet activation and platelet microaggregation that cannot be assayed, imaged or pharmacologically characterised with the conventional methods.

\section{Acknowledgements}

We are grateful to Science Foundation Ireland (SFI) for the financial support to MWR and MJSM. CM is a SFI stokes lecturer. APM is supported by the EC FP7 NAMDIATREAM (NMP-2009-246479) research project.

\section{References}

1 B. Furie and B. C. Furie, N. Engl. J. Med., 2008, 359, 938949.

2 A. D. Michelson, J. Thromb. Thrombolysis, 2003, 16, 7-12.

3 J. Rivera, M. L. Lozano, L. Navarro-Núñez and V. Vicente, Haematologica, 2009, 94, 700-711.

4 Z. M. Ruggeri and G. L. Mendolicchio, Circ. Res., 2007, 100, 16731685 .

5 P. Needleman, S. Moncada, S. Bunting, J. R. Vane, M. Hamberg and B. Samuelsson, Nature, 1976, 261, 558-560.

6 G. V. Born, Br. J. Haematol., 1966, 12, 37-38.

7 G. Sawicki, E. Salas, J. Murat, H. Miszta_Lane and M. W. Radomski, Nature, 1997, 386, 616-619.

8 G. Davi and C. Patrono, N. Engl. J. Med., 2007, 357, 2482-2494.

9 Z. M. Ruggeri, Nat. Med. (N. Y.), 2002, 8, 1227-1234.

10 M. D. Linden and D. E. Jackson, Int. J. Biochem. Cell Biol., 2010, 42, 1762-1766.

11 J. R. O’Brien, J. Clin. Pathol., 1961, 14, 140-149.

12 G. V. R. Born, Nature, 1962, 194, 972-979.

13 M. H. Kroll, J. D. Hellums, L. V. McIntire, A. I. Schafer and J. L. Moake, Blood, 1996, 88, 1525-1541.

14 D. C. Cardinal and R. J. Flower, J. Pharmacol. Methods, 1980, 3, 135-158.

15 J. W. Smith, S. R. Steinhubl, A. M. Lincoff, J. C. Coleman, T. T. Lee, R. S. Hillman and B. S. Coller, Circulation, 1999, 99, 620-625.

16 J. J. Zwaginga, K. S. Sakariassen, G. Nash, M. R. King, J. W. Heemskerk, M. Frojmovic and M. F. Hoylaerts, J. Thromb. Haemostasis, 2006, 4, 2716-2717.

17 P. Harrison, A. L. Frelinger, M. I. Furman and A. D. Michelson, Thromb. Res., 2007, 120, 323-336.

18 L. G. Pedvis, T. Wong and M. M. Frojmovic, Thromb. Haemostasis, 1988, 59, 323-328.

19 K. Konstantopoulos, S. Kukreti and L. V. McIntire, Adv. Drug Delivery Rev., 1998, 33, 141-164.

20 S. K. Kundu, E. J. Heilmann, R. Sio, C. Garcia, R. M. Davidson and R. A. Ostgaard, Semin. Thromb. Hemostasis, 1995, 21, 106-112.

21 C. P. Hayward, P. Harrison, M. Cattaneo, T. L. Ortel and A. K. Rao, Platelet physiology subcommittee of the scientific and standardization committee of the international society on thrombosis and haemostasis, J. Thromb. Haemostasis, 2006, 4, 312-319.

22 G. M. Podda, P. Bucciarelli, F. Lussana, A. Lecchi and M. Cattaneo, J. Thromb. Haemostasis, 2007, 5, 2393-2398.

23 Z. Xia and M. M. Frojmovic, Biophys. J., 1994, 66, 2190-2201.

24 M. M. Frojmovic, Ann. Biomed. Eng., 2008, 36, 528-533.

25 H. R. Baumgartner and C. Haudenschild, Ann. N. Y. Acad. Sci., 1972, 27, 22-36.

26 K. S. Sakariassen, S. R. Hanson and Y. Cadroy, Thromb. Res., 2001, 104, 149-174.

27 K. S. Sakariassen, R. Muggli and H. R. Baumgartner, Methods Enzymol., 1989, 169, 37-70.

28 A. O. Maree and D. J. Fitzgerald, Circulation, 2007, 115, 21962207.

29 A. López Farré, J. Tamargo, P. Mateos-Cáceres, L. Azcona and C. Macaya, Pharm. Res., 2010, 27, 2365-2373.

30 D. J. Angiolillo, M. Ueno and S. Goto, Circ. J., 2010, 74, 597-607.

31 D. W. Inglis, K. J. Morton, J. A. Davis, T. J. Zieziulewicz, D. A. Lawrence, R. H. Austin and J. C. Stur, Lab Chip, 2008, 8, 925-931.

32 E. Gutierrez, B. G. Petrich, S. J. Shattil, M. H. Ginsberg, A. Groisman and A. Kasirer-Friede, Lab Chip, 2008, 8, 1486-1495.

33 K. B. Neeves, S. F. Maloney, K. P. Fong, A. A. Schmaier, M. L. Kahn, L. F. Brass and S. L. Diamond, J. Thromb. Haemostasis, 2008, 6, 2193-2201.

34 D. P. Sarvepalli, D. W. Schmidtke and M. U. Nollert, Ann. Biomed. Eng., 2009, 37, 1331-1341.

35 S. W. Schneider, S. Nuschele, A. Wixforth, C. Gorzelanny, A. Alexander-Katz, R. R. Netz and M. F. Schneider, Proc. Natl. Acad. Sci. U. S. A., 2007, 104, 7899-7903.

36 N. J. Kent, L. Basabe-Desmonts, G. Meade, B. D. MacCraith, B. G. Corcoran, D. Kenny and A. J. Ricco, Biomed. Microdevices, 2010, 12, 987-1000.

37 L. Tran, J. Farinas, L. Ruslim-Litrus, P. B. Conley, C. Muir, K. Munnelly, D. M. Sedlock and D. B. Cherbavaz, Anal. Biochem., 2005, 341, 361-368. 
38 C. J. Ku, T. D'Amico Oblak and D. M. Spence, Anal. Chem., 2008, 80, 7543-7548.

39 S. F. Maloney, F. L. Brass and S. L. Diamond, Integr. Biol. (Camb)., 2010, 2, 183-192.

40 F. J. Tovar-Lopez, G. Rosengarten, E. Westein, K. Khoshmanesh, S. P. Jackson, A. Mitchell and W. S. Nesbitt, Lab Chip, 2010, 10, 291-302.

41 D. Bazou, M. J. Santos-Martinez, C. Medina and M. W. Radomski, Br. J. Pharmacol., 2011, 162, 1577-1589.

42 T. Goerge, F. Kleinerüschkamp, A. Barg, E. M. Schnaeker, V. Huck, M. F. Schneider, M. Steinhoff and S. W. Schneider, Thromb. Haemostasis, 2007, 98, 283-286.

43 N. J. Kent, S. O’Brien, L. Basabe-Desmonts, G. R. Meade, B. D. MacCraith, B. G. Corcoran, D. Kenny and A. J. Ricco, IEEE Trans. Biomed. Eng., 2011, 58, 826-830.

44 G. Sauerbrey, Z. Phys., 1959, 155, 206-222.

45 S. Sinn, L. Muller, H. Drechsel, M. Wandel, H. Northoff, G. Ziemer, H. P. Wendel and F. K. Gehring, Analyst, 2010, 135, 2930-2938.

46 T. Matsuda, A. Kishida, H. Ebato and Y. Okahata, ASAIO J., 1992, 38, M171-M173.
47 K. Kawakami, Y. Harada, M. Sakasita, H. Nagai, M. Handa and Y. Ikeda, ASAIO J., 1993, 39, M558-M560.

48 M. Muratsugu, A. D. Romanschin and M. Thompson, Anal. Chim. Acta, 1997, 342, 23-29.

49 M. Rodahl, F. Hook, A. Krozer, P. Brzezinski and B. Kasemo, Rev. Sci. Instrum., 1995, 66, 3924-3930.

50 C. Fredriksson, S. Khilman, B. Kasemo and D. M. Steel, J. Mater. Sci.: Mater. Med., 1998, 9, 785-788.

51 C. Fredriksson, S. Kihlman, M. Rodahl and B. Kasemo, Langmuir, 1998, 14, 248-251.

52 M. J. Santos-Martinez, Ph.D. Thesis, Trinity College Dublin, 2009.

53 M. J. Santos-Martínez, C. Medina, A. Prina-Mello, J. Conroy, S. P. Samuels, Y. Volkov and M. W. Radomski, Kardiochirurgia $i$ Torakochirurgia Polska, 2010, 7, 365-375.

54 N. Weber, H. P. Wendel and J. Kohn, J. Biomed. Mater. Res., Part A, 2005, 72, 420-427.

55 J. Fatisson, Y. Merhi and M. Tabrizian, Langmuir, 2008, 24, 3294 3299.

56 J. Fatisson, S. Mansouri, D. Yacoub, Y. Merhi and M. Tabrizian, J. R. Soc., Interface, 2011, 8, 988-997. 